\title{
Bayesian approach to comparing the left ventricular volume in myocardial infarction and in normal cases
}

\author{
A.T. Hernowo, M.D. Ph.D.
}

\begin{abstract}
Introduction. Post-contrast delayed-enhancement MRI helps to show the extent of myocardial infarction (MI), as well as allowing the morphometric analysis of the heart structure, e.g., left ventricular volume (LVV). Here the author deployed Bayesian implementation of statistical inference to determine the difference between the LVV in MI cases and in normal controls. Methods. Sixty-seven MI patients and thirty-three controls from the EMIDEC dataset challenge were used. These were acquired at the University Hospital of Dijon (France). A cardiologist with 10 years of experience in cardiology and MRI and a biophysicist with 20 years of experience of cardiovascular MRI supervised the acquisition and annotated the images. ITK-Snap was used to extract the LVV and Bayesian inference were used to investigate the data. Results. Patients with MI were younger than the controls $\left(58.3 \pm 11.5\right.$ vs. $66.5 \pm 12.9$ years; $\left.B_{10}=17.9\right)$. With age taken into consideration, the patients showed larger $L V V$ relative to the controls $\left(128.1 \pm 46.3\right.$ vs. $83.4 \pm 24.4 \mathrm{~cm}^{3}$; post-hoc $\mathrm{BF}_{10}=$ 12663.8). Summary. Using Bayesian approach, we can conclude decisively that there is volumetric difference or remodeling in individuals with $\mathrm{MI}$.
\end{abstract}

Index Terms-myocardial infarction, magnetic resonance imaging, Bayesian statistics, morphometry

\section{INTRODUCTION}

Magnetic resonance imaging (MRI) has been providing non-invasive cardiac imaging and guiding physicians in the management of myocardial infatcion. The widely accepted practice is the application of delayed enhancement postgadolinium injection T1-weighted imaging [1], with optional T2-weighted acquisition to visualize any edematous tissue. The post-contrast delayed-enhancement MRI (DE-MRI) helps to show the extent of myocardial infarction (MI), as well as allowing the morphometric analysis of the heart structure, e.g., left ventricular volume (LVV).

While group study of either MI or the LVV may have been widely done in various combinations, the approach to the statistical inference are relatively uniform, involving frequentist null-hypothesis significance testing. Nonetheless, Bayesian inference has been getting traction in recent years. [2], [3] There are three main reasons why Bayesian approach is better than the frequentist one: (1) quantifiable evidence in favor of both the null and the alternate hypothesis; (2) prior knowledge utilization; and (3) tangible values of the evidence as more data are accumulated and computed. [4]

Here the author deployed Bayesian implementation of statistical inference to determine the difference between the LVV in MI cases and in normal controls. To show the strength of the evidence supporting either the null or alternate hypothesis, Bayes factor $\left(\mathrm{BF}_{10}\right)$ will be computed.

A.T. Hernowo is with Department of Anatomy, Faculty of Medicine Universitas Islam Indonesia, Indonesia, e-mail: dr.hernowo@uii.ac.id (Corresponding author).

\section{Methods}

Sixty-seven MI patients and thirty-three controls from the automatic Evaluation of Myocardial Infarction from DelayedEnhancement Cardiac MRI (EMIDEC) dataset challenge were used. [5] These were acquired at the University Hospital of Dijon (France). The data were completely anonymized and their acquisition and handling were abiding to the local ethical committee regulation. Since these were compiled retrospectively and completely stripped off their administrative information, it was not necessary to do the process to have ethical approval number.

Siemens MRI scanners (Area (1.5 T) and Skyra (3T)) were used to image the heart during cardiovascular exam with no specific protocol. Short-axis slices of the DE-MRI have been extracted in a retrospective study. The acquisitions taken during breath-hold (ECG-gated), 10 minutes after gadoliniumbased contrast agent injection. Resulting MR images were arranged from left ventricular base to apex. Pixel spacing between $1.25 \times 1.25 \mathrm{~mm}^{2}$ and $2 \times 2 \mathrm{~mm}^{2}$, slice thickness $8 \mathrm{~mm}$, and distance between slices between 8 and $13 \mathrm{~mm}$. The images were provided in NIFTI format. Three screenshot samples ventricular wall and chamber annotation from the controls and the patients group can be seen in the following figure.

A cardiologist with 10 years of experience in cardiology and MRI and a biophysicist with 20 years of experience of cardiovascular MRI supervised the acquisition and annotated the images as seen in figure 1. ITK-SNAP was used to extract the LVV and Bayesian inference were used to investigate the data.

\section{RESULTS}

Mean LVVs of the patients and the controls are described in table 1.

TABLE I

LEFT VENTRICULAR VOLUME IN CONTROLS AND PATIENTS GROUP.

\begin{tabular}{lcccccc}
\hline & & & & & \multicolumn{2}{c}{$95 \%$ CI } \\
\cline { 6 - 7 } Group & $\mathrm{N}$ & Mean & SD & SE & Lower & Upper \\
\hline Controls & 33 & 83.37 & 24.41 & 4.25 & 74.72 & 92.03 \\
Patients & 67 & 128.13 & 46.29 & 5.66 & 116.83 & 139.42 \\
\hline
\end{tabular}

Group comparison using Bayesian version of t-test for independent samples revealed that the LVV of the patients is more likely to be different from and larger than the controls $\left(\mathrm{BF}_{10}=12,663.21\right.$; error $\left.2.5 \cdot 10^{-7} \%\right)$. Effect size $(\delta)$ parameter estimation is illustrated below.

The larger LVV of the patients is attributed with a large effect size $(\delta=-1.041 ; 95 \% C I-1.492--0.595-1.041$; 

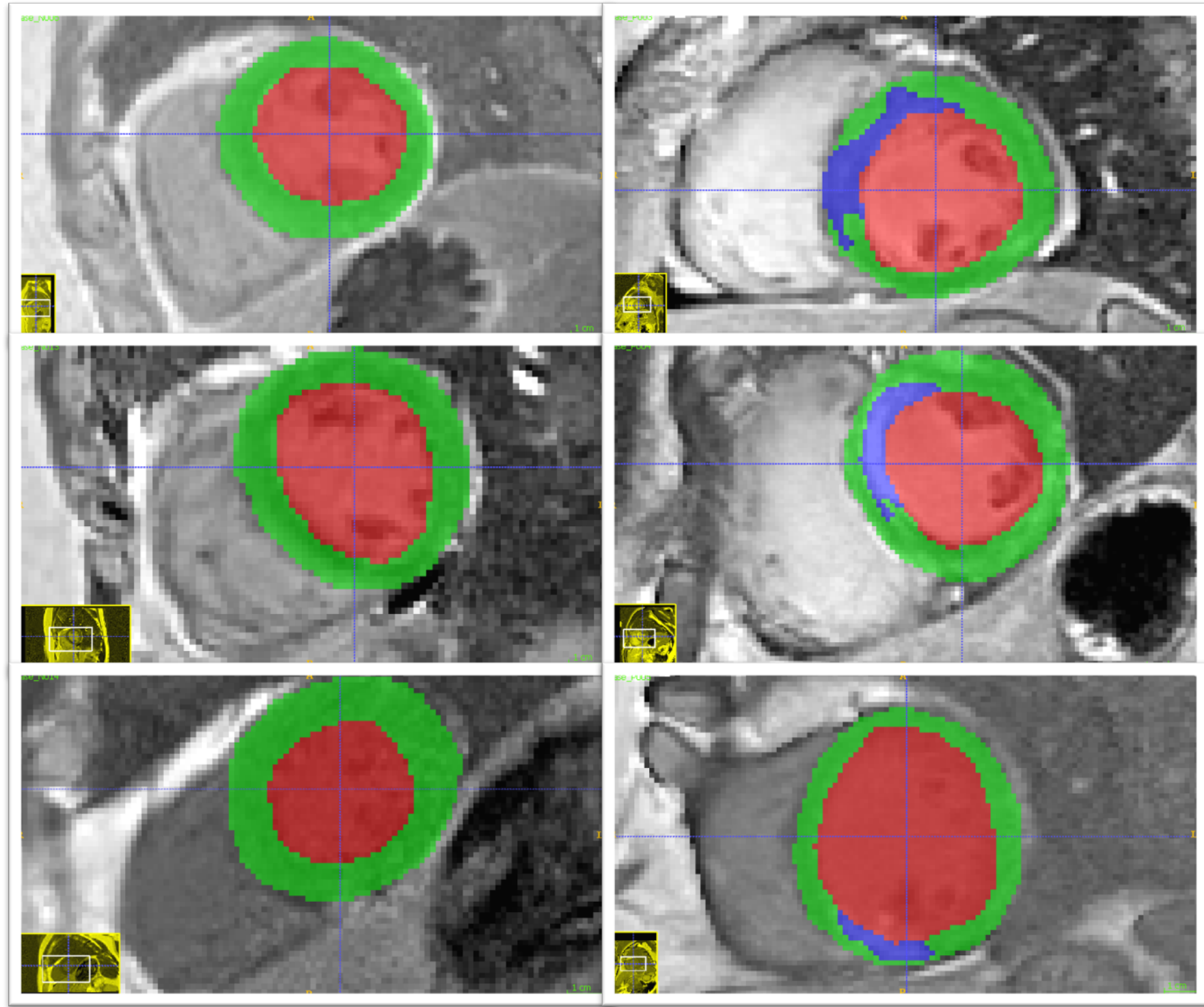

Fig. 1. DE-MRI image of the left ventricle. Three random sample left ventricle cross-sectional (mid way between the base and the apex) images are displayed. The controls are on the left side and the patients on the right. The ventricular chamber is annotated with red, while the wall with green and blue colors. Green indicates normally perfused wall, whereas blue indicates the infarction zone.

95\%CI -1.492 - -0.595). To check the robustness of the Bayes factor, varying priors are used and the result can be seen in figure 2 .

The $\mathrm{BF}_{10}$ value varies narrowly between 12,663 and 13,507, consistently above 10,000 . To see how the Bayes factor vary as more data points were computed, a sequential analysis was done and illustrated in figure 3.

The evidential flow for the alternative hypothesis $\left(\mathrm{H}_{1}\right)$ was relatively stable between $10^{3}-10^{4}$ and hence categorized as an extreme evidence supporting $\mathrm{H}_{1}$. To be prudent, we compared the mean age between the two groups. The patients were found to be younger than the controls $(58.3 \pm 11.5$ vs. $66.5 \pm 12.9$ years; $\mathrm{BF}_{10}=17.9$; error $=6.777 \cdot 10^{-5} \%$ ). Based from the age comparison, it was necessary to take into account when comparing the LVV between the two groups. With age taken into consideration, the patients still showed larger LVV relative to the controls (post-hoc $\mathrm{BF}_{10}=12,663.84$ ).

\section{DiscusSION}

The left ventricular volume has been known to increase in patients with myocardial infarction, even as early as 4 weeks after the event. [6], [7], [8], [9] The findings have been confirmed in many studies despite using different modalities to measure the LVV, among which are echocardiography and MRI.

Nonetheless, those reports invariably applied frequentist approach to test their $\mathrm{H}_{0}$. It eventually resulted in the acceptance or negation of their $\mathrm{H}_{0}$, using $p$-value. The $p$-value itself is proven invaluable to simply nullified the $\mathrm{H}_{0}$, but it does not provide any measure of the strength of the evidence backing up or against the alternate hypothesis $\left(\mathrm{H}_{1}\right)$ nor the $\mathrm{H}_{0}$. p-value is able to show how unlikely the data support the $\mathrm{H}_{0}$, but it cannot show how the data support the $\mathrm{H}_{1}$. One may, however, argue that the magnitude of difference can be stated as the 


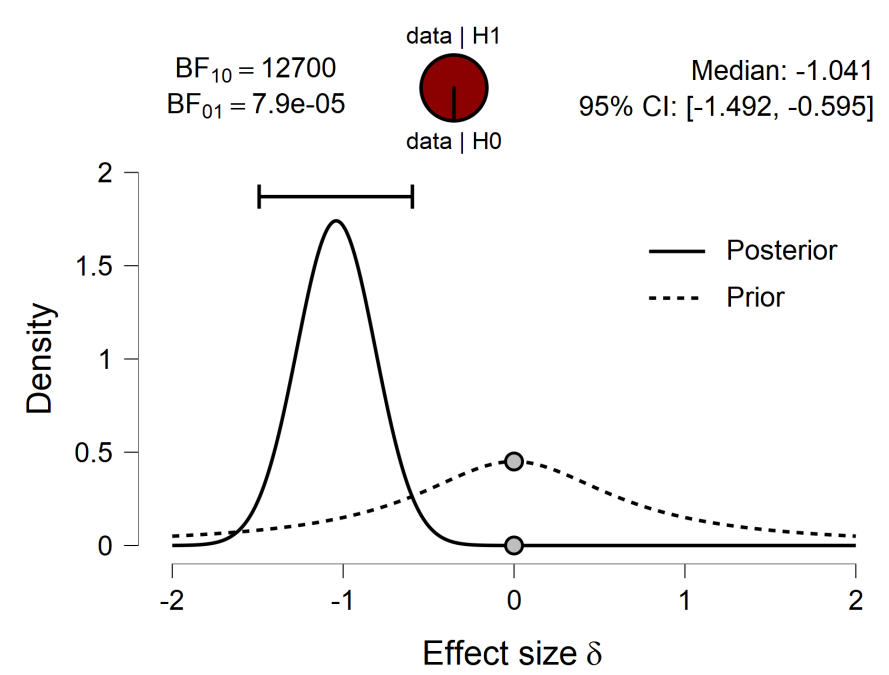

Fig. 2. Bayesian two-sample t-test for the parameter $\delta$.

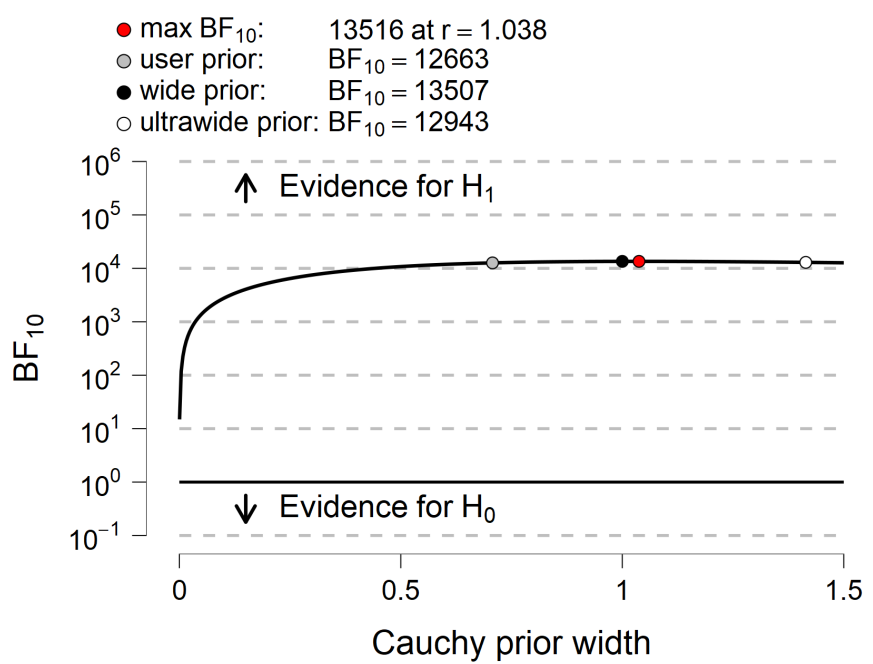

Fig. 3. The Bayes factor robustness plot.

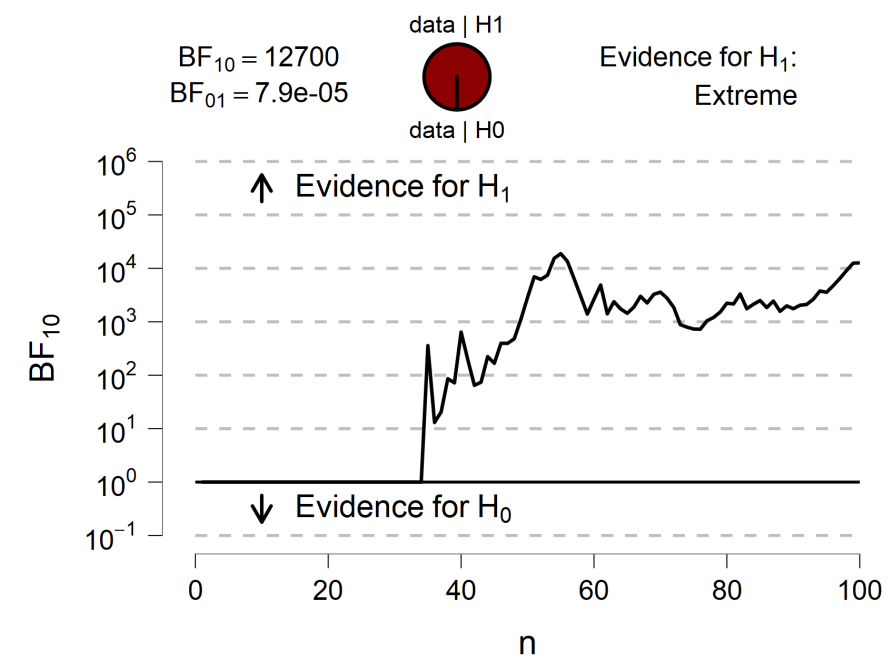

Fig. 4. Sequential analysis of the Bayes factor. effect size $(\delta)$. While $\delta$ may indicate whether, for example, the mean difference between two groups is small (negligible) or large, $\delta$ does not indicate the likelihood of the data points to be under or supporting a given hypothesis. Bayes factor on the other hand, indicate a scalar of evidential strength for either the $\mathrm{H}_{0}$ or $\mathrm{H}_{1}$.

In this report, the author discovered that the LVV in patients is greater than in controls with a $\mathrm{BF}_{10}$ of 12,663 . Quantitatively, we can say that data are 12,663 times more likely to support the $\mathrm{H}_{1}$ rather than the $\mathrm{H}_{0}$. In other words, it is extremely likely ( $>10,000$ times more likely) that any LVV measurement in patients with MI will be different (or in this case, greater) from the healthy controls (figure 5).

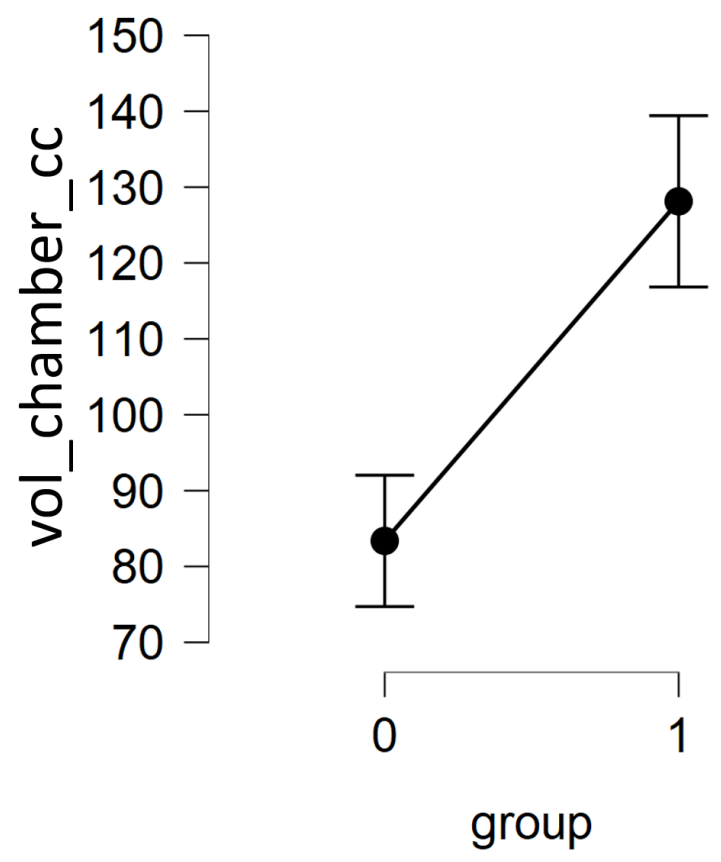

Fig. 5. Mean left ventricular chamber volume comparison between controls ("0") and patients ("1").

With this kind of results, the author can be cautiously confident that any measurement of the LVV in a patient with MI using this MRI-based volumetry method, will yield a value that will be generally greater than the one of normal individuals of comparable age. Therefore, this finding that the LVV is increased with MI can be considered a general truth. The author recommends more frequent deployment of Bayesian techniques in health or clinical studies to reach quantifiable evidence to build more robust stepping stones for even more further studies.

\section{REFERENCES}

[1] B. Kendziora, H. Stier, P. Schlattmann, and M. Dewey, "MRI for measuring therapy efficiency after revascularisation in ST-segment elevation myocardial infarction: a systematic review and meta-regression analysis," BMJ Open, vol. 10, no. 9, 2020.

[2] M. Andrews and T. Baguley, "Prior approval: The growth of Bayesian methods in psychology," 2013.

[3] J. Vandekerckhove, J. N. Rouder, and J. K. Kruschke, "Bayesian methods for advancing psychological science," 2018. 
medRxiv preprint doi: https://doi.org/10.1101/2021.09.26.21264134; this version posted September 28, 2021. The copyright holder for this

preprint (which was not certified by peer review) is the author/funder, who has granted medRxiv a license to display the preprint in perpetuity.

It is made available under a CC-BY-NC 4.0 International license .

[4] J. van Doorn, D. van den Bergh, U. Böhm, F. Dablander, K. Derks, T. Draws, A. Etz, N. J. Evans, Q. F. Gronau, and J. M. Haaf, "The JASP guidelines for conducting and reporting a Bayesian analysis," Psychonomic Bulletin \&amp; Review, vol. 28, no. 3, pp. 813-826, 2021.

[5] A. Lalande, Z. Chen, T. Decourselle, A. Qayyum, T. Pommier, L. Lorgis, E. de la Rosa, A. Cochet, Y. Cottin, and D. Ginhac, "Emidec: a database usable for the automatic evaluation of myocardial infarction from delayedenhancement cardiac MRI," Data, vol. 5, no. 4, p. 89, 2020.

[6] R. W. Jeremy, R. A. Hackworthy, G. Bautovich, B. F. Hutton, and P. J. Harris, "Infarct artery perfusion and changes in left ventricular volume in the month after acute myocardial infarction," Journal of the American College of Cardiology, vol. 9, no. 5, pp. 989-995, 1987.

[7] G. A. Lamas and M. A. Pfeffer, "Increased left ventricular volume following myocardial infarction in man," American heart journal, vol. 111, no. 1, pp. 30-35, 1986.

[8] R. W. Jeremy, K. C. Allman, G. Bautovitch, and P. J. Harris, "Patterns of left ventricular dilation during the six months after myocardial infarction," Journal of the American College of Cardiology, vol. 13, no. 2, pp. 304$310,1989$.

[9] P. Gaudron, C. Eilles, I. Kugler, and G. Ertl, "Progressive left ventricular dysfunction and remodeling after myocardial infarction. Potential mechanisms and early predictors." Circulation, vol. 87, no. 3, pp. 755-763, 1993. 